Trabajos originales 


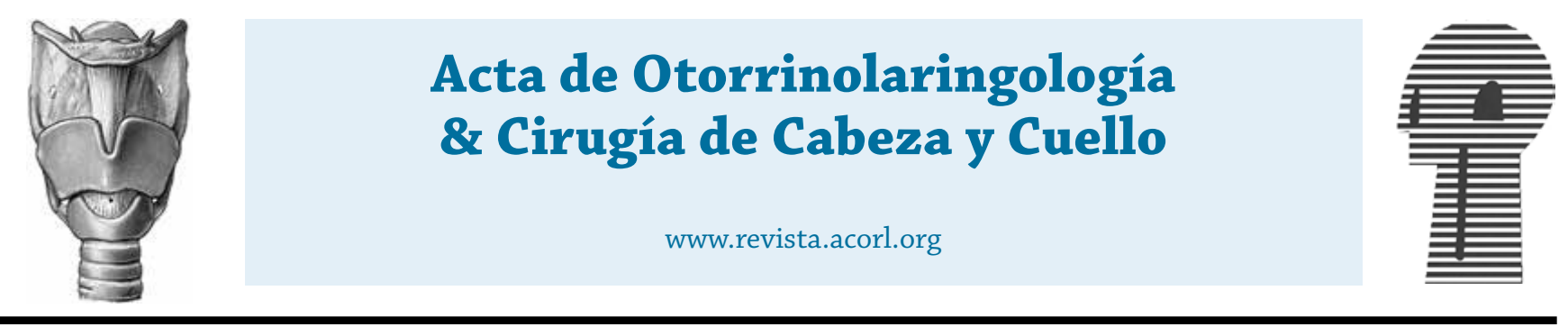

\title{
Caracterización de la disfonía en la población infantil en un centro de laringología en Bogotá, Colombia
}

\section{Characterization of dysphonia in pediatric age in a laryngology center in Bogotá, Colombia}

\author{
Elisa Gutiérrez Gómez*, Danielle Bastida Rosas*, María Carolina Bermúdez**, Luis Humberto Jiménez* \\ * Unidad de Otorrinolaringología y Cirugía Maxilofacial, Hospital Universitario San Ignacio. Bogotá, Colombia. \\ ** Jefe Unidad de Otorrinolaringología, Fundación Neumológica Colombiana. Bogotá, Colombia.
}

Forma de citar: Gutiérrez-Gómez E. Bastida-Rosas D. Bermúdez MC. Jiménez LH. Caracterización de la disfonía en la población infantil en un centro de laringología en Bogotá, Colombia. Acta otorrinolaringol. cir. cabeza cuello. 2020;48(2):134-141. Doi:10.37076/acorl.v48i2.486

\section{INFORMACIÓN DEL ARTÍCULO}

\section{Historia del artículo:}

Recibido: 23 de Abril de 2020

Evaluado: 16 de Junio de 2020

Aceptado: 20 de Junio de 2020

Palabras clave (DeCS):

Disfonía, larínge, voz, niño.

\section{RESUMEN}

Introducción: la disfonía en edad infantil está presente entre el $6 \%$ al $23 \%$ de los niños entre los 4-12 años y su estudio supone un reto diagnóstico y terapéutico debido a que los procedimientos actualmente utilizados requieren de una completa colaboración del paciente. En Colombia, hasta el momento, no se ha realizado un estudio sobre la caracterización de las principales patologías que causan disfonía en niños. Los hallazgos son similares a los reportados en la literatura mundial; sin embargo, los nódulos vocales no son la principal causa de disfonía en niños en nuestra población como se había reportado hasta ahora. Es por esto por lo que el objetivo del presente estudio es proporcionar los resultados de nuestra casuística en cuanto a patologías de cuerdas vocales en la población pediátrica con disfonía crónica (mayor a 4 semanas), que asisten a consulta externa de laringología en el Hospital Universitario San Ignacio en Bogotá, Colombia, con el fin de conocer su frecuencia y características asociadas. Materiales y métodos: se realiza un estudio retrospectivo de tipo descriptivo, en el que se analizaron historias clínicas y estroboscopias realizadas a pacientes menores de 18 años con síntomas vocales crónicos

\section{Correspondencia:}

Elisa Gutiérrez Gómez

E-mail: egutierrez@husi.org.co

Dirección: carrera 7.a 40-69; oficina 712, Bogotá 110111, Colombia.

Teléfono celular:3203452869 
(mayores a 4 semanas), que asistieron a consulta de laringología en los últimos 10 años. Posteriormente se realizó un análisis estadístico y descriptivo de las mismas. Resultados: se seleccionaron 141 pacientes; de estos, $73 \%$ tuvo lesiones de ambos pliegues vocales, 9,9\% lesiones unilaterales y $16,3 \%$ disfonía por tensión muscular primaria. La mayoría de las lesiones fueron adquiridas, con leve predominio en el pliegue vocal izquierdo y sexo masculino. La patología más prevalente fue el sulcus vocalis $(17,37 \%)$, seguido de la disfonía por tensión muscular primaria y nódulos vocales $(13,22 \%)$. El 41,06 \% de los pacientes se catalogaron como abusadores de la voz, y dentro de este grupo, los nódulos vocales fueron más frecuentes. Discusión: se encontró una predominancia del sulcus vocalis, lo cual difiere con la literatura en la que, tradicionalmente, se describen los nódulos; en este estudio se encontró que estos estaban relacionados con el abuso de la voz en la mitad de los pacientes. Adicionalmente se identificó una relación entre la disfonía por tensión muscular y el canto, además de síntomas de reflujo gastroesofágico en algunos niños. Conclusiones: este es el primer estudio de su tipo en Colombia y el segundo en Latinoamérica. Los hallazgos en general son consistentes con la literatura, siendo la única diferencia que la primera causa de disfonía fue el sulcus vocalis. Se encontró una relación entre los nódulos y los quistes con el abuso de la voz, lo que apoya la teoría de la naturaleza adquirida de estos últimos.

\section{ABSTRACT}

Key words (MeSH):

Dysphonia, larynx voice, child.
Introduction: dysphonia is present in $6 \%$ to $23 \%$ of children between $4-12$ years of age and its study suppose a diagnostic and therapeutic challenge because the procedures currently used require a complete collaboration from the patient. In Colombia, a study on the characterization of the main pathologies that cause dysphonia in children has not been carried out so far. The findings are like those reported in the world literature, however, vocal nodules are not the main cause of dysphonia in children in our population as reported so far. The objective of this study is to report our stroboscopic findings in children with chronic dysphonia (more than 4 weeks) that attend to the laryngology service of the San Ignacio University Hospital, in Bogotá, Colombia, and compare them with the international literature. Materials and methods: A retrospective descriptive study was conducted. Medical records and stroboscopies of patients younger than 18 years with chronical voice symptoms (greater than 4 weeks), that assisted to a laryngology consultation at the San Ignacio University Hospital, in Bogotá, Colombia within the last 10 years were analyzed. A descriptive statistical analysis was performed. Results: 141 patients were selected, $73 \%$ had bilateral vocal fold lesions, $9.9 \%$ unilateral lesions and $16.3 \%$ had primary muscle tension dysphonia. Most of the lesions were acquired, with a slight predominance of lesion on the left vocal fold and in males. The most common finding was vocal sulcus $(13.37 \%)$, followed by muscle tension dysphonia and vocal nodules (13.2 $\%)$. A $41.06 \%$ of patients referred vocal abuse, and within this group, vocal nodules where most prevalent. Discussion: there was a predominance of vocal sulcus, this differs from the literature in which vocal nodules are the most frequent finding. In this study, vocal nodules where related to chronic voice abuse, which was referred by half of the patients. A relation between muscle tension dysphonia and singing was also observed, an important number of patients referred gastroesophageal reflux symptoms as well. Conclusions: this is the first study in Colombia and the second one in Latin America of its kind. The results are generally consistent with the literature, but the first cause of dysphonia in our study is vocal sulcus, as opposed to vocal nodules which are typically the most common cause. There was a relation between chronic voice abuse, vocal nodules and cysts, this supports the theory that cysts are acquired lesions and not congenital. 


\section{Introducción}

Hasta un tercio de la población general presenta a lo largo de su vida patologías de la voz, las cuales se presentan en igual proporción entre niños y adultos, y sus causas varían según el grupo de edad (1). Asimismo, la disfonía en edad infantil está presente en el $6 \%$ al $23 \%$ de los niños entre los 4-12 años (2), y su estudio supone un reto diagnóstico y terapéutico debido a que los procedimientos actualmente utilizados, a pesar de ser poco invasivos, son incómodos y requieren de una completa colaboración del niño, así como de una completa disposición por parte del profesional para realizarlos.

En general, los desórdenes de la voz se categorizan en tres grandes grupos: sindrómicos, secundarios a lesiones estructurales y funcionales. Los nódulos vocales están ubicados dentro del grupo de las lesiones estructurales y siguen siendo considerados como la primera causa de disfonía en los niños (2-6); no obstante, en la práctica diaria se encuentra una variedad de causas de igual importancia, pero menos reconocidas, como la parálisis de pliegues vocales, las membranas laríngeas y los quistes, entre otras. En la actualidad, a nivel latinoamericano y mundial, contamos con poca bibliografía concerniente a la disfonía en niños (2), razón por la cual este estudio es el primero que caracteriza los hallazgos estroboscópicos y su prevalencia en la población infantil con disfonía crónica en Colombia.

\section{Materiales y métodos}

En este estudio retrospectivo de tipo descriptivo se revisaron las historias clínicas y las estroboscopias realizadas a los pacientes menores de 18 años, que consultaron por presentar síntomas vocales crónicos (definidos como síntomas persistentes por más de 4 semanas), en la consulta externa por un subespecialista en laringe, entre los años 2014 a 2019, en la consulta de laringología, en el Hospital Universitario San Ignacio en la ciudad de Bogotá, Colombia.

Se excluyeron todos los casos con historias clínicas incompletas, niños con traqueostomía o estenosis de laringe comprobada y pacientes con síntomas respiratorios infecciosos agudos altos o bajos. Se realizó una evaluación de la fonación y la laringe con estroboscopio Storz PULSAR ${ }^{\mathrm{TM}}$ modelo 20140020 con cámara HD Storz Image 1 Hub y telelaringoscopio de 70 grados Storz. Las imágenes fueron obtenidas y analizadas a 1,0 ciclos/segundo.

Las variables de interés fueron clasificadas en características sociodemográficas, hallazgos principales y secundarios en la estroboscopia, historia de tratamiento previo, patologías asociadas, realización de nasolaringoscopia previa y síntomas concomitantes asociados. El análisis se realizó utilizando el programa STATA ${ }^{\circledR}$ (statistics/data análisis, edición especial 15.1). Las variables categóricas fueron descritas por medio de frecuencias y porcentajes, mientras que las numéricas mediante medidas de tendencia central y dispersión.

El trabajo de estudio fue aprobado por el Comité de investigaciones de la Pontificia Universidad Javeriana y del
Hospital Universitario San Ignacio, de acuerdo con los principios de la Declaración de Helsinki.

\section{Resultados}

\section{Selección de pacientes}

De 2014 a 2019 se evaluaron un total de 255 pacientes, de los cuales se seleccionaron 142 niños entre los 4 y 18 años, mientras que 113 fueron retirados del estudio según los criterios de exclusión. Posteriormente, de los 142 pacientes seleccionados, se excluyó a un niño de 4 años dado que no se logró realizar la estroboscopia por poca colaboración del paciente. Finalmente, se analizaron 141 pacientes, para un total de 242 pliegues vocales (PV) (Figura 1); de estos, el 57,04 $\%$ fueron hombres. El $50 \%$ de los pacientes estudiados tenían entre 7 y 14 años, la edad media para la realización de la estroboscopia fue de 10,8 años con una desviación estándar (DE) de 3,84 años.

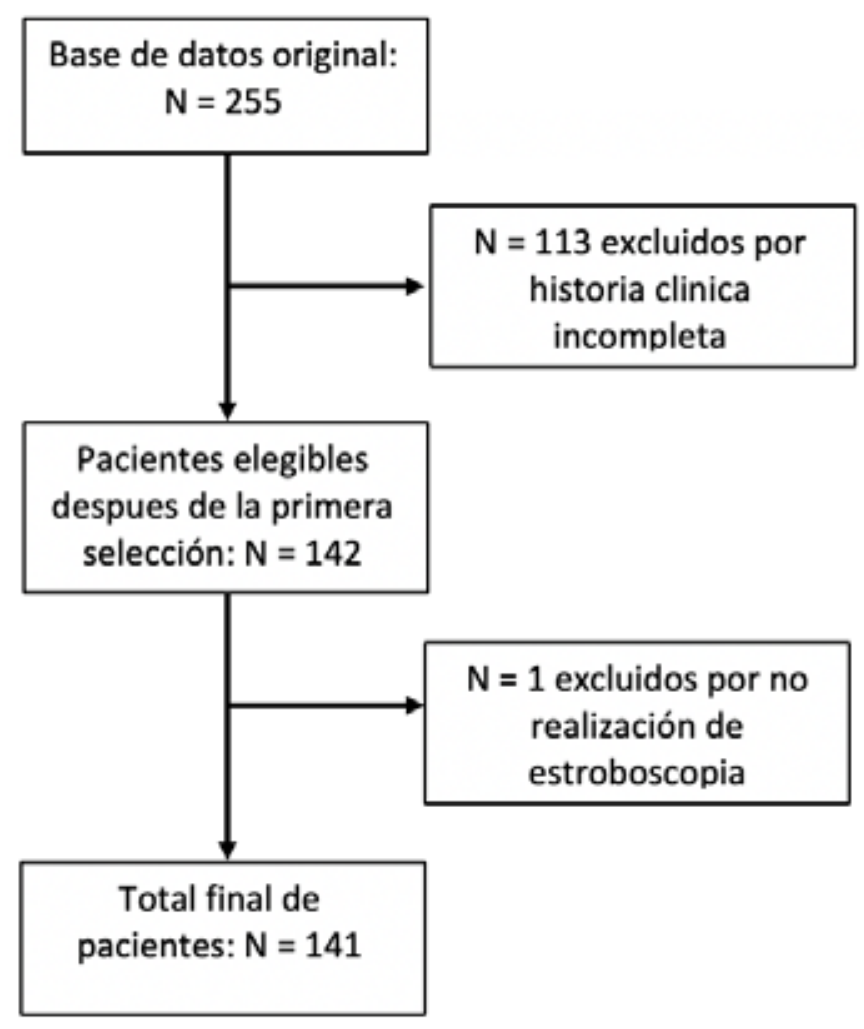

Base de datos original: $\mathrm{N}=255 \mid \mathrm{N}=113$ excluidos por historia clínica incompleta | Pacientes elegibles después de la primera selección: $N$ $=142 \mid \mathrm{N}=1$ excluidos por no realización de estroboscopia $\mid$ Total final de pacientes: $\mathrm{N}=141$

Figura 1. Algoritmo de selección de pacientes.

\section{Hallazgos generales}

De los 141 pacientes, 103 (73\%) tenían lesiones de ambos PV para un total de $206 \mathrm{PV}, 14$ (9,9\%) presentaron lesiones unilaterales y $23(16,3 \%)$ padecían de disfonía por tensión 
muscular primaria. Adicionalmente se encontró que la mayoría de las lesiones eran de naturaleza adquirida, lo cual fue evidente en el PV derecho con un 65,96\% de las lesiones y $68,79 \%$ de las lesiones del PV izquierdo.

Del total de estroboscopias analizadas, se encontró que el hallazgo más prevalente fue el sulcus vocalis, el cual se encontró en el 17,37 \% de la totalidad de las estroboscopias. El segundo hallazgo más frecuente fue la disfonía por tensión muscular primaria evidenciada en un 16,31\% de los casos, seguida de los nódulos vocales en un total de $13,12 \%$ y, finalmente, la cuarta patología más prevalente fueron los quistes, para un total de 10,99\%. (Tabla 1).

Hallazgos en pliegue vocal izquierdo frente al pliegue vocal derecho

Con respecto a la PV izquierdo, se encontró como principal patología el sulcus vocalis para un total de 27 casos $(19,14$ $\%)$, de los cuales $16(11,35 \%)$ corresponden a sulcus vocalis tipo I, $10(7,09 \%)$ corresponden a sulcus vocalis tipo II y 1 $(0,71 \%)$ corresponde a sulcus vocalis tipo III. En el PV derecho estos hallazgos varían, ya que se encuentra un porcentaje menor de sulcus vocalis tipo I con respecto a PV izquierdo, para un total de $5,67 \%$ y hay un porcentaje mayor de sulcus vocalis tipo II $(9,93 \%)$ para pliegue vocal derecho frente al PV izquierdo. Adicionalmente, en PV derecho no se evidenciaron casos de sulcus vocalis tipo III (Figura 2).
Tabla 1. Hallazgos principales encontrados en pliegues vocales

\begin{tabular}{|l|c|c|}
\hline Patologías de pliegues vocales & Frecuencia & Porcentaje \\
\hline Sulcus vocalis & 49 & $17,37 \%$ \\
\hline Tensión muscular primaria & 46 & $16,31 \%$ \\
\hline Nódulos & 37 & $13,12 \%$ \\
\hline Quiste & 31 & $10,99 \%$ \\
\hline Trauma por lesión contralateral & 22 & $7,80 \%$ \\
\hline Pseudoquiste & 20 & $7,09 \%$ \\
\hline Otros & 14 & $4,96 \%$ \\
\hline Ninguno & 14 & $4,96 \%$ \\
\hline Quiste frente a pseudoquiste & 9 & $3,19 \%$ \\
\hline Lesiones estructurales mínimas & 8 & $2,83 \%$ \\
\hline de la cubierta & & \\
\hline Edema subepitelial & 8 & $2,83 \%$ \\
\hline Membrana laríngea & 6 & $2,12 \%$ \\
\hline Edema de Reinke & 6 & $2,12 \%$ \\
\hline Laringitis & 4 & $1,41 \%$ \\
\hline Puentes de mucosa & 2 & $0,70 \%$ \\
\hline Estenosis subglótica & 2 & $0,70 \%$ \\
\hline Parálisis unilateral & 1 & $0,35 \%$ \\
\hline Parálisis bilateral & 1 & $0,35 \%$ \\
\hline Papilomas & reportado & $0,35 \%$ \\
\hline Pólipo & & \\
\hline
\end{tabular}

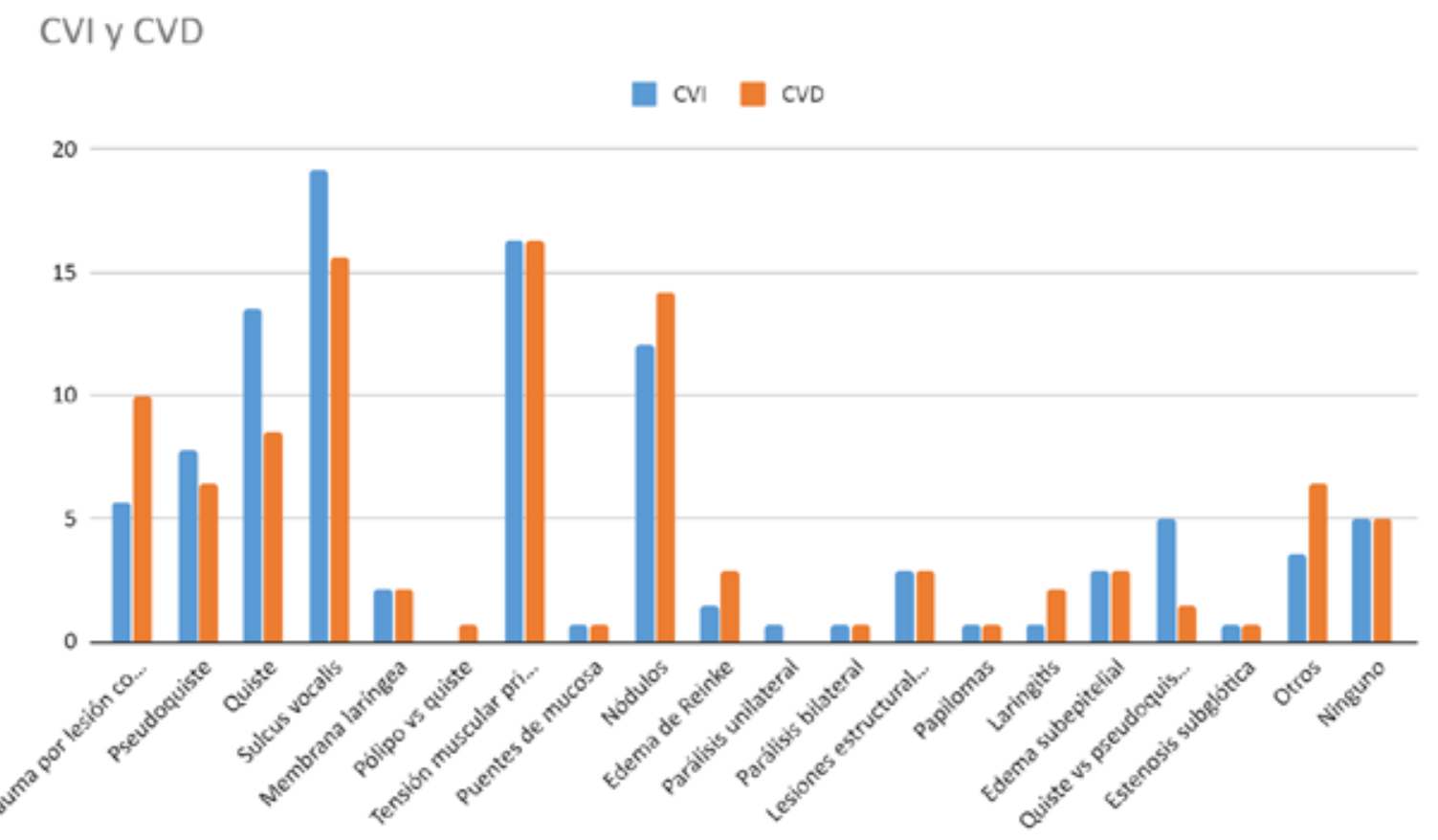

PVI | PVD | Trauma por lesión contralateral | Pseudoquiste |Quiste | Sulcus vocalis | Membrana laríngea | Pólipo frente a quiste | Tensión muscular primaria | Puentes de mucosa | Nódulos | Edema de Reinke | Parálisis unilateral | Lesiones estructurales mínimas de la cubierta | Papilomas | Laringitis | Edema subepitelial | Quiste frente a pseudoquiste | Estenosis subglótica | Otros | Ninguno

Figura 2. Hallazgos principales encontrados en los pliegues vocales izquierdo y derecho.

PVI: pliegue vocal izquierdo; PVD: pliegue vocal derecho. 
Con respecto a los nódulos vocales, se encontró una disminución discreta en el número de casos cuando se comparó con el PV derecho (12,06 \% frente a $14,81 \%$, respectivamente). Otro hallazgo relevante fue el trauma por lesión contralateral, el cual se encontró en el 5,67\% de los PV izquierdos y 9,93\% de los PV derechos (Figura 2).

En cuanto a los quistes, se encuentra que hay un aumento importante de la presencia de estos en el PV izquierdo en comparación con el derecho $(13,48 \%$ frente a $8,51 \%)$, además de los casos de quiste frente a pseudoquiste encontrados en el $4,96 \%$ de los casos en el PV izquierdo y $1,42 \%$ en el PV derecho y un único caso en el PV derecho, en el que no fue posible diferenciar entre un quiste y un pólipo (Figura 2).

Otros hallazgos importantes, pero menos prevalentes que se encontraron fueron las membranas laríngeas, el edema de Reinke, laringitis, el edema subepitelial, entre otros. Se encontraron hallazgos clasificados como "otros" hasta en un 3,55\% y 4,96\% para PV izquierdo y derecho, respectivamente, dentro de que se encuentran las cicatrices, la secreción mucoide y los bordes irregulares. En la Figura 3 se muestran dos ejemplos de hallazgos encontrados en las estroboscopias.

\section{Otros hallazgos}

El 51,06\% $(\mathrm{N}=72)$ de los pacientes se catalogaron como abusadores de voz; en este grupo de niños, la patología que se ve con más frecuencia son los nódulos vocales, los cuales se observaron en $24 \mathrm{PV}(33,34 \%)$, siendo ligeramente más prevalente en el PV derecho $(18,06 \%)$, seguido de los sulcus, los cuales se encontraron en 22 PV (31,9\%). Asimismo, se observó una elevada prevalencia de quistes $(29,16 \%)$, encontrándose dos veces más en el PV izquierdo en comparación con el derecho (14 niños frente a 7 niños, respectivamente), teniendo en cuenta que en 6 casos adicionales no fue posible diferenciar quiste y pseudoquiste, por lo que la prevalencia podría ser más alta. Finalmente, en estas estroboscopias se evidenció una prevalencia igual en el trauma contralateral de PV $\mathrm{y}$ en el pseudoquiste, que corresponde al 18,45\% (Tabla 2).

También se encontró en nuestra población que el 23,4\% $(\mathrm{N}=33)$ de los pacientes se encuentran en clases de canto y el 19,5 \% referían síntomas o diagnóstico de reflujo gastroesofágico. En los pacientes que cantan, el diagnóstico más frecuente fue el sulcus vocalis tipo I (36,36\%), seguido de la disfonía por tensión muscular primaria $(33,33 \%)$.

Con respecto a los hallazgos secundarios, la tensión muscular secundaria se presentó con una frecuencia de $62 \%(90$ pacientes), laringitis/faringitis que corresponde al 37,6\% (53 casos), y en menor medida pseudosulcus traumático e incompetencia glótica, con una prevalencia de 2,13\% y 7,8 $\%$, respectivamente.

Con respecto a tratamientos y exámenes previos, encontramos que en nuestra población únicamente el 4,96 \% de los pacientes tenían reportada una nasofibrolaringoscopia previa y el $19,29 \%$ de los pacientes habían tenido terapia de voz previa; sin embargo, cabe anotar que, en el $36,43 \%$ se desconocía si habían recibido terapia vocal.

\section{Análisis y discusión}

La literatura reporta que la disfonía en edad infantil está presente en el $6 \%$ al $23 \%$ de los niños entre los 4 y 12 años (2). A nivel latinoamericano, Martins y colaboradores han sido los únicos en caracterizar la disfonía en niños (2), por lo que este aparece como el segundo trabajo de este tipo a nivel continental y el primero en Colombia hasta la fecha.

La edad media de los pacientes fue de 10,8 años, con una $\mathrm{DE}$ de 3,8 años. La mayoría de las patologías encontradas son adquiridas y de predominio masculino, lo que concuerda con lo reportado en la literatura $(2,3)$. En nuestro estudio se encontró una prevalencia de $57 \%$ de hombres frente al $43 \%$ de mujeres, similar a lo que reportan Angelillo y colaboradores (7), quienes tuvieron una muestra mayor con un total
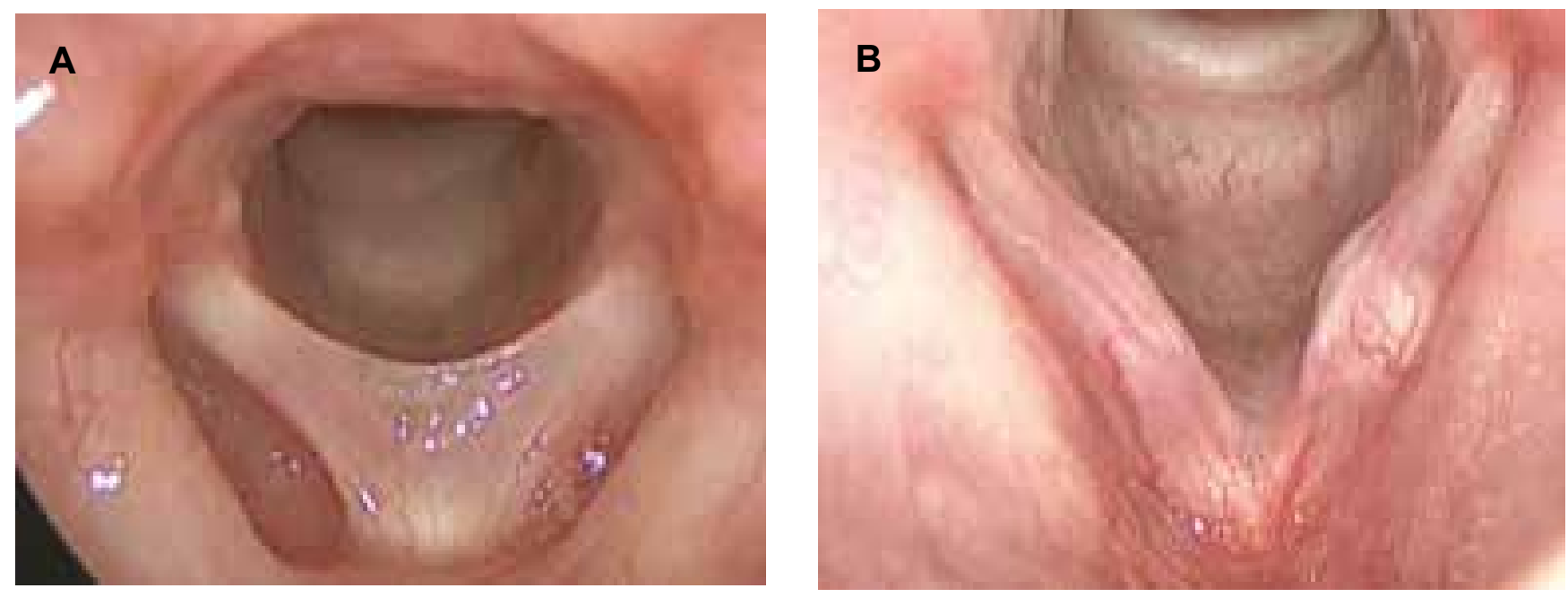

Figura 3. Ejemplo de dos estroboscopias realizadas en población pediátrica. A. Hallazgo de membrana laríngea. B. Lesión estructural mínima de la cubierta del pliegue vocal izquierdo. 
Tabla 2. Hallazgos principales encontrados en pacientes que abusan de la voz

\begin{tabular}{|c|c|c|}
\hline Patologías de pliegue vocal & $\begin{array}{c}\text { Pliegue vocal derecho (Número de } \\
\text { pacientes y } \% \text { ) }\end{array}$ & $\begin{array}{l}\text { Pliegue vocal izquierdo (Número de } \\
\text { pacientes y \%) }\end{array}$ \\
\hline Trauma por lesión contralateral & $10(13,89)$ & $4(5,56)$ \\
\hline Pseudoquiste & $7(9,72)$ & $7(9,72)$ \\
\hline Quiste & $7(9,72)$ & $14(19,44)$ \\
\hline Sulcus vocalis tipo I & $3(4,17)$ & $8(11,11)$ \\
\hline Sulcus vocalis tipo II & $7(9,72)$ & $4(5,56)$ \\
\hline Sulcus vocalis tipo III (bolsa) & No hay casos & $1(1,39)$ \\
\hline Pólipo & No hay casos & No hay casos \\
\hline Membrana laríngea & $1(1,39)$ & $1(1,39)$ \\
\hline Pólipo frente a quiste & No hay casos & No hay casos \\
\hline Tensión muscular primaria & $8(11,11)$ & $9(12,5)$ \\
\hline Puentes de mucosa & No hay casos & No hay casos \\
\hline Nódulos & $13(18,08)$ & $11(15,28)$ \\
\hline Edema de Reinke & $2(2,78)$ & $2(2,78)$ \\
\hline Parálisis unilateral & No hay casos & No hay casos \\
\hline Parálisis bilateral & No hay casos & No hay casos \\
\hline Lesiones estructurales mínimas de la cubierta & $4(5,56)$ & $4(5,56)$ \\
\hline Papilomas & No hay casos & No hay casos \\
\hline Laringitis & No hay casos & No hay casos \\
\hline Otros & $3(4,17)$ & $3(4,17)$ \\
\hline Ninguno & $4(5,56)$ & No hay casos \\
\hline Edema subepitelial & $1(1,39)$ & No hay casos \\
\hline Quiste frente a pseudoquiste & $2(2,78)$ & $4(5,56)$ \\
\hline Estenosis subglótica & No hay casos & No hay casos \\
\hline
\end{tabular}

de 178 casos. En otros estudios reportados en la literatura, como el de Martins y colaboradores (1) y el de Conelly y colaboradores $(2,8)$, se reporta una predominancia incluso mayor en hombres hasta del $64 \%$ frente al $36 \%$ en mujeres. Con respecto a la frecuencia de las patologías, se encontró una discrepancia entre lo descrito en los diferentes artículos, los cuales afirman que la patología más frecuente que afecta a los niños con disfonía crónica son los nódulos (3-5). En este estudio, esta fue la tercera causa después del sulcus vocalis y la tensión muscular primaria, con una prevalencia de $17,37 \%$ para sulcus vocalis, $16,31 \%$ para tensión muscular primaria y $13,12 \%$ para nódulos vocales, lo que podría suponer un subdiagnóstico de los sulcus vocalis teniendo en cuenta las limitaciones en la realización de la estroboscopia antes mencionadas, ya que estos no pueden ser visualizados adecuadamente con lente de nasolaringoscopia flexible.

Adicionalmente se encontró que la mayoría de los niños abusaban de la voz (51,06\%), lo que concuerda con lo que se ha reportado hasta el momento en la literatura, donde se ha encontrado el abuso de la voz como la principal causa de disfonía en niños (8). Sin embargo, en nuestro estudio, la prevalencia de patología en los PV en niños que no abusan de la voz es aún muy alta $(48,94 \%)$, puesto que se encontró un importante número de patologías congénitas que pueden pasarse por alto al momento de realizar el diagnóstico.
Dentro de los hallazgos en niños que abusan de la voz se identifica el nódulo vocal como la primera causa de disfonía, pudiéndose observar en aproximadamente $1 / 3$ de los pacientes. Esta asociación ya había sido descrita desde la década de 1960 por múltiples autores, quienes, además, atribuyen esto al hecho de que la mayoría de los niños gritan durante el juego (9-12). Más recientemente Akif y colaboradores reportaron una prevalencia similar a la de este estudio en la población infantil (12). De igual forma, es importante anotar que la presencia de quistes en los abusadores vocales es más elevada en comparación con los que no abusan de la voz, lo que apoya la teoría de varios autores de que estas lesiones son de etiología adquirida y no congénita (13).

Los nódulos vocales tienden a disminuir su tamaño durante la adolescencia debido a la acción de las hormonas sexuales $(2,14,15)$, principalmente en los hombres, en quienes es más evidente este cambio; por esta razón, el tratamiento de elección en este grupo de edad es la terapia de voz antes que el tratamiento quirúrgico (2).

En relación con los quistes, se encontró como la cuarta causa más frecuente dentro de esta población de pacientes; no obstante, llama la atención que hubo una confusión en el diagnóstico de esa patología con pseudoquistes y pólipos, lo que pudiese indicar un subdiagnóstico en este hallazgo. 
En cuanto al grupo de edad, en los niños de la primera infancia (entre 0-5 años), las patologías más encontradas fueron los sulcus vocalis y las membranas laríngeas, lo que podría indicar una prevalencia mayor de patologías congénitas en este grupo de edad. Por otra parte, el grupo con mayor número de pacientes fue el de los niños entre 6 a 11 años (infancia), con un total de 72 niños (50,70 \%). En este grupo, la principal patología encontrada fueron los quistes con una prevalencia del $18,05 \%$, seguida por los nódulos vocales con una prevalencia de $15,97 \%$, lo que concuerda con la literatura en la que se evidencia que en este grupo de edad prevalecen las patologías adquiridas (8). Finalmente, en la adolescencia (12 a 18 años) la patología más frecuente fueron los pseudoquistes $(37,6 \%)$, seguidos de la tensión muscular primaria $(15,2 \%)$; esto podría indicar que posiblemente están relacionadas con el tiempo de exposición al trauma y el abuso vocal.

La tensión muscular ocupa el segundo puesto de prevalencia en el grupo general de niños; sin embargo, en el grupo de los niños que refieren canto, esta patología ocupa el segundo lugar. Lo anterior atribuirse a la falta de entrenamiento vocal actual o previo y comportamientos que causan trauma vocal como la sobre articulación, el aclaramiento vocal y un aumento excesivo del tono de voz (16).

De los pacientes estudiados, el 82,98\% $(\mathrm{N}=117)$ no contaban con nasofibrolaringoscopia previa, y de los 24 pacientes con nasolaringoscopia previa únicamente el 29,19 $\%$ concordaba con los hallazgos en la estroboscopia, lo que apoya la importancia de realizar un estudio completo y detallado con estroboscopia en los pacientes pediátricos con síntomas crónicos de la voz, con el fin de establecer el adecuado tratamiento y control.

Dentro de los factores de riesgo asociados se encontraron, principalmente, el refllujo gastroesofágico, antecedente de canto y el abuso vocal (Figura 4). En cuanto al reflujo gastroesofágico, 19,15\% $(\mathrm{N}=27)$ de los pacientes refirió síntomas de reflujo gastroesofágico y dentro de los hallazgos en estroboscopia únicamente 4 presentaban signos sugestivos de reflujo gastroesofágico; sin embargo, no se realizaron pruebas específicas para definir la presencia de enfermedad por reflujo gastroesofágico (ERGE), lo que plantea el interrogante sobre la relación del reflujo gastroesofágico con disfonía en niños y la necesidad de iniciar tratamiento empírico.

Según la guía de práctica clínica de disfonía de la Academia Americana de Otorrinolaringología y Cirugía de Cabeza y Cuello (AAO-HNSF, por sus siglas en inglés), se recomienda no dar tratamiento empírico para reflujo gastroesofágico en casos de disfonía con síntomas de reflujo gastroesofágico sin antes visualizar la laringe, y se recomienda prescribirlos en pacientes con disfonía y signos de laringitis crónica (1). No obstante, la evidencia es controvertida, si bien hay artículos que demuestran que entre los pacientes con disfonía y reflujo hay tendencia hacia la mejoría con el manejo médico $(17,18)$, Una revisión sistemática de Cochrane encontró una respuesta similar al placebo cuando se da tratamiento médico en estos pacientes; sin embargo, la calidad de la información científica es pobre, lo que impide dar una conclusión fuerte (19). Adicionalmente se considera que, si bien la visualización directa de la laringe puede indicar signos que sugieren reflujo gastroesofágico, su especificidad es muy baja y, por lo tanto, el diagnóstico no debe basarse en estos resultados (1).

Se encontraron lesiones menos frecuentes como la papilomatosis laríngea, parálisis de pliegues vocales, estenosis subglótica y puentes de mucosa, que corresponden al $0,35 \%$ de los casos cada una. Los papilomas se encontraron únicamente en 1 caso; sin embargo, es de gran importancia tenerlos en cuenta en el diagnóstico diferencial de los pacientes pediátricos con disfonía, teniendo en cuenta que se trata de la neoplasia benigna más importan-

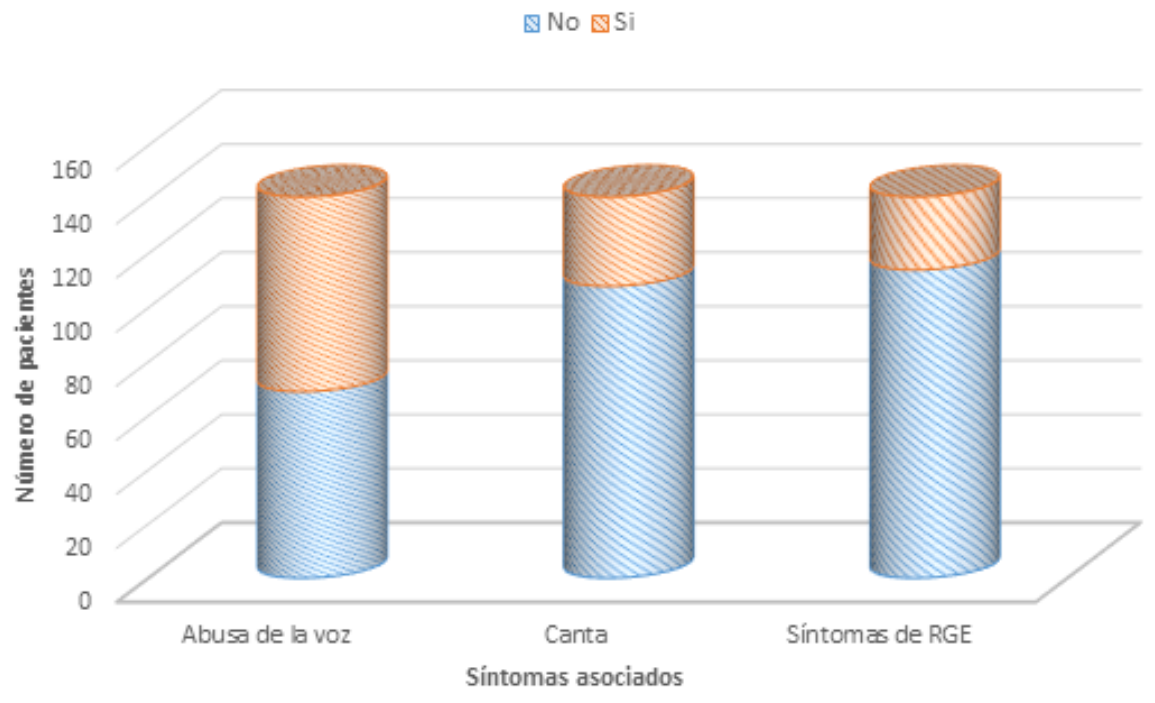

Número de pacientes | No | Sí | Abusa de la voz | Canta | Síntomas de ERGE | Síntomas asociados

Figura 4. Principales factores de riesgo asociados encontrados en los pacientes estudiados. 
te de la laringe por su aparición temprana, ya que tiende a reaparecer a pesar del manejo y, en ocasiones, con compromiso de la vía aérea (2).

\section{Conclusiones}

Este es el primer estudio de su tipo en Colombia y de los pocos en la literatura médica latinoamericana. Los hallazgos en general son consistentes con aquellos vistos en otras partes del mundo, siendo la principal diferencia que en esta población se encontró como primera causa de disfonía a los sulcus vocalis y no a los nódulos, como es típicamente descrito. La tensión muscular fue identificada como una causa prevalente de disfonía en los niños que cantan, con una prevalencia elevada de nódulos dentro de la población pediátrica que abusa de la voz. Notablemente, los quistes también se vieron asociados a la población abusadora vocal, lo que apoya la teoría de que estos son de tipo adquiridos y no congénitos.

Este estudio posee varias limitaciones, entre ellas el tamaño poblacional, el cual es pequeño en comparación a otros similares a nivel mundial; adicionalmente, en algunos pacientes no fue posible la adquisición de los datos completos dada la naturaleza retrospectiva del estudio. Es necesario realizar un estudio prospectivo, en el que pueda evaluarse una muestra mayor, así como los factores de riesgo asociados, de manera que pueda tenerse una mejor caracterización de los pacientes en nuestra población.

\section{Conflicto de interés}

No presenta ningún conflicto de interés

\section{REFERENCIAS}

1. Stachler RJ, Francis DO, Schwartz SR, Damask CC, Digoy GP, Krouse HJ, et al. Clinical Practice Guideline: Hoarseness (Dysphonia)(Update). OtolaryngolNeckSurg.2018;158(3):409426. https//doi.org/10.1177/0194599817751031

2. Martins RHG, Hidalgo Ribeiro CB, Fernandes de Mello BMZ, Branco A, Tavares ELM. Dysphonia in Children. J Voice. 2012;26(5):674.e17-674.e6.74E20. https//doi.org/10.1016/j. jvoice.2012.03.004

3. Choi SS, Zalzal GH. Voice disorders in children. En: Cummings Otolaryngology, Head \& Neck Surgery. 6.a ed. Filadelfia: Elsevier; 2015. p. 3133-41.

4. Gray SD, Smith ME, Schneider H. VOICE DISORDERS IN CHILDREN. Pediatr Otolaryngol. 1996;43(6):1357-1384. https//doi.org/10.1016/s0031-3955(05)70523-x
5. Levitsky SE. Hoarseness. En: Primary pediatric care. 4.a ed. St. Louis: Mosby; 2001. p. 1156.

6. Yang $\mathrm{J}, \mathrm{Xu} \mathrm{W}$. Characteristics of Functional Dysphonia in Children. J Voice. 2020;34(1):156.e1-156.e4. https//doi. org/10.1016/j.jvoice.2018.07.027

7. Angelillo N, Di Costanzo B, Angelillo M, Costa G, Barillari MR, Barillari U. Epidemiological study on vocal disorders in paediatric age. J Prev Med Hyg. 2008;49(1):1-5.

8. Connelly A, Clement WA, Kubba H. Management of dysphonia in children. J Laryngol Otol. 2009;123(6):642-7. https//doi. org/10.1017/S0022215109004599

9. Toohill RJ. The Psychosomatic Aspects of Children With Vocal Nodules. Arch Otolaryngol. 1975 Oct;101:5. https//doi. org/10.1001/archotol.1975.00780390005002

10. Wilson DK. Children with Vocal Nodules. J Speech Hear Disord. 1961;26(1):19-26.

11. Batza EM. Vocal Cord Nodules in Children: Pathogenesis, Clinical Manifestations, Therapy. Clin Pediatr (Phila). 1970;9(1):14-6. https//doi.org/10.1177/000992287000900105

12. Kay NJ. Vocal nodules in children - aetiology and management. J Laryngol Otol. 1982;96:731-6. https//doi.org/10.1017/ s0022215100093051

13. Martins RHG, do Amaral HA, Tavares ELM, Martins MG, Gonçalves TM, Dias NH. Voice Disorders: Etiology and Diagnosis. J Voice. 2016;30(6):761.e1-761.e9. https//doi. org/10.1016/j.jvoice.2015.09.017

14. Nardone HC, Recko T, Huang L, Nuss RC. A Retrospective Review of the Progression of Pediatric Vocal Fold Nodules. JAMA Otolaryngol Neck Surg. 2014;140(3):233. https//doi. org/10.1001/jamaoto.2013.6378

15. Nunes RB1, Behlau M, Nunes MB, Paulino JG. Clinical diagnosis and histological analysis of vocal nodules and polyps. Braz J Otorhinolaryngol. 2013;79:434-40. https//doi. org/10.1001/jamaoto.2013.6378

16. Teachey JC, Kahane JC, Beckford NS. Vocal mechanics in untrained professional singers. J Voice. 1991;5(1):51-6.

17. Qua CS, Wong CH, Gopala K, Goh KL. Gastro-oesophageal reflux disease in chronic laryngitis: prevalence and response to acid-suppressive therapy. Aliment Pharmacol Ther. 2007;25(3):287-295. https//doi.org/10.1111/j.13652036.2006.03185.x

18. Kahrilas PJ, Shaheen NJ, Vaezi MF. American Gastroenterological Association Medical Position Statement on the Management of Gastroesophageal Reflux Disease. Gastroenterology. 2008;135(4):1383-1391.e5. https//doi. org/10.1053/j.gastro.2008.08.045

19. Hopkins C, Yousaf U, Pedersen M. Acid reflux treatment for hoarseness. Cochrane Database Syst Rev. 2006;(1):CD005054. https//doi.org/10.1002/14651858.CD005054.pub2 